\title{
Evolução temporal das componentes geomagnéticas horizontal e vertical na região da Anomalia Magnética do Atlântico Sul
}

\author{
Caprara, B. B. ${ }^{*}$, UNIPAMPA; Cruz, M. F., UNIPAMPA; Herdies, G. R., UNIPAMPA; Frigo, E., UNIPAMPA.
}

Copyright 2018, SBGf - Sociedade Brasileira de Geofísica

Este texto foi preparado para a apresentação no VIII Simpósio Brasileiro de Geofísica, Salinópolis, 18 a 20 de setembro de 2018. Seu conteúdo foi revisado pelo Comitê Técnico do VIII SimBGf, mas não necessariamente representa a opinião da SBGf ou de seus associados. É proibida a reprodução total ou parcial deste material para propósitos comerciais sem prévia autorização da SBGf.

\section{Resumo}

A região da Anomalia Magnética do Atlântico Sul (AMAS) é caracterizada pela baixa intensidade total do campo geomagnético e pelo alto fluxo de partículas eletricamente carregadas (PEC). Em razão da contribuição de componentes não dipolares, o padrão de variação temporal e espacial dos elementos geomagnéticos nesta região difere daquele esperado pelo modelo de campo geomagnético exclusivamente dipolar. Neste trabalho são estudadas as variações da intensidade geomagnética total $(F)$, horizontal $(H)$, vertical (Z) e das razões H/F e Z/F entre 1900 e 2015 para a região influenciada pela AMAS. Os resultados indicam uma tendência de decréscimo de $\mathrm{F}, \mathrm{H}$ e $\mathrm{H} / \mathrm{F}$ ao longo do tempo. A persistência deste comportamento nos próximos anos pode favorecer a ocorrência de fenômenos associados as PEC na região.

\begin{abstract}
The region of the South Atlantic Magnetic Anomaly (SAMA) is characterized by low values of geomagnetic total intensity and high fluxes of electrically charged particles (ECP). Due to the contribution of non-dipole components, the pattern of temporal and spatial variations of the geomagnetic elements in this area differs from that expected by the exclusively dipole geomagnetic field model. In this work, we investigated the variations of the geomagnetic total intensity $(F)$, horizontal $(H)$ vertical (Z) geomagnetic components and the ratios $\mathrm{H} / \mathrm{F}$ and $\mathrm{Z} / \mathrm{F}$ for the interval between 1900 and 2015 for the SAMA influenced region. The results indicate that $\mathrm{F}, \mathrm{H}$ and $\mathrm{H} / \mathrm{F}$ are decreasing during the last century. The persistence of this behavior in the next years may favor the occurrence of phenomena associated with the ECP in the investigated region.
\end{abstract}

\section{Introdução}

O campo magnético da Terra, que é caracterizado por uma configuração predominantemente dipolar, é gerado majoritariamente no interior terrestre através do mecanismo de geodínamo. A intensidade geomagnética total $(F)$, para cada posição e para cada instante de tempo, é resultante das contribuições relativas da intensidade horizontal $(H)$ e da intensidade vertical $(Z)$. Considerando um modelo de campo geomagnético exclusivamente dipolar, os maiores valores de $\mathrm{F}$ seriam observados nas regiões polares, onde a contribuição de $\mathrm{Z}$ é máxima e a contribuição de $\mathrm{H}$ é mínima. Por outro lado, os menores valores de $\mathrm{F}$ seriam observados na região equatorial, onde a contribuição de $\mathrm{H}$ é máxima e a contribuição de Z é mínima.

O campo geomagnético funciona como um escudo natural que protege a Terra contra efeitos indesejáveis associados a penetração de partículas eletricamente carregadas (PEC) provenientes do espaço (Campbell, 2003). Entre estes efeitos estão os danos em componentes eletrônicos de satélites, problemas em redes de transmissão de energia elétrica, e até mesmo malefícios à saúde humana.

Para que uma partícula eletricamente carregada penetre a barreira imposta pelo campo magnético, é necessário que sua energia seja superior a um valor denominado rigidez magnética de corte $(R C)$. Os valores de $R C$ são mais altos em regiões onde os valores de $\mathrm{H}$ são mais altos e, são mais baixos onde os valores de $\mathrm{H}$ são mais baixos (Smart \& Shea, 2009). Assumindo a configuração dipolar do campo geomagnético, espera-se que os valores de $\mathrm{RC}$ diminuam da região equatorial em direção as regiões polares, seguindo a configuração esperada para a intensidade geomagnética horizontal.

Entretanto, em algumas regiões da Terra, como na região da Anomalia Magnética do Atlântico Sul (AMAS), caracterizada por baixos valores de $F$, existe uma significativa contribuição de componentes não dipolares para o campo geomagnético (Hartmann \& Pacca, 2009). Adicionalmente, na região da AMAS, são observados altos fluxos de elétrons, prótons, nêutrons e radiação gama (Grigoryan et al., 2008). No entanto, apesar dos pontos de máximo fluxo de partículas seguirem uma trajetória de deriva para o oeste similar à do ponto de mínimo de F, eles não são coincidentes. Esta diferença pode estar associada a influência da parcela não dipolar do campo geomagnético nesta região, que altera os valores de $\mathrm{H}, \mathrm{Z}$ e $\mathrm{F}$ em relação aqueles esperados pelo modelo dipolar.

Neste trabalho, são investigadas as contribuições das componentes geomagnéticas horizontal e vertical para a intensidade total, na região da América do Sul mais influenciada pela AMAS, considerando o período compreendido entre os anos de 1900 e 2015. 


\section{Dados e Métodos de Análise}

A área de estudo abrange toda a região sul e parte das regiões sudeste e centro-oeste do Brasil, além de partes dos territórios de Uruguai, Argentina, Paraguai e Bolívia e, uma região do Oceano Atlântico (Figura 1). Na área de estudo foram definidos pontos constituindo um grid com resolução espacial de 1 grau de latitude por 1 grau de longitude. Para cada ponto foram obtidas as séries temporais de $\mathrm{H}, \mathrm{Z}$ e $\mathrm{F}$.

As séries temporais foram obtidas a partir do modelo International Geomagnetic Reference Field (IGRF). Este modelo, que representa o campo geomagnético de origem interna à Terra e suas variações temporais e espaciais, é construído a partir do ajuste de dados observacionais utilizando-se uma função de harmônicos esféricos (Merrill et al., 1998). A implementação computacional do modelo foi realizada a partir da rotina igrf12.f, escrita em linguagem Fortran, disponibilizada pela International Association of Geomagnetism and Aeronomy na página da National Oceanic and Atmospheric Administration dos Estados Unidos (https://www.ngdc.noaa.gov/lAGA/vmod/igrf.html).

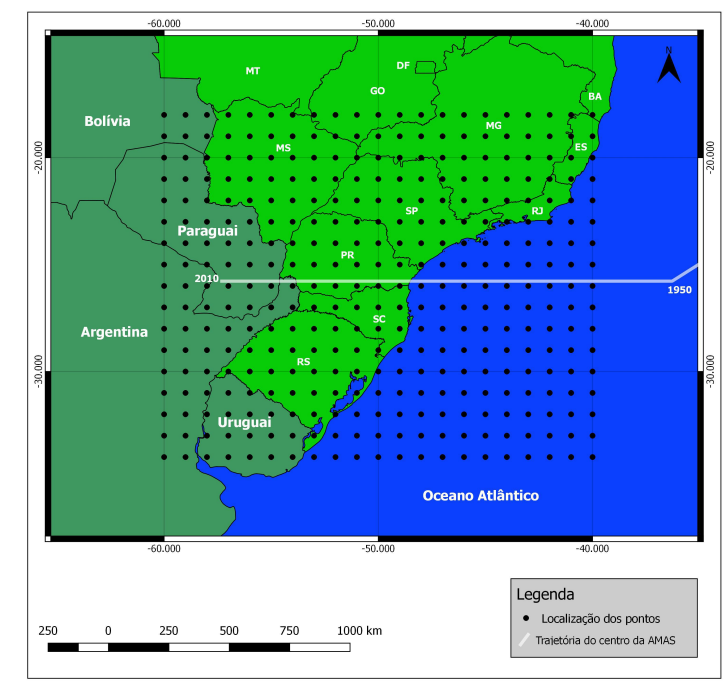

Figura 1- Mapa de localização da área de estudo indicando as posições dos pontos analisados e a trajetória aproximada do centro da AMAS.

A partir dos dados de $\mathrm{H}, \mathrm{Z}$ e $\mathrm{F}$ para cada ponto, são calculadas as contribuições relativas de $\mathrm{H}$ para $\mathrm{F}(\mathrm{H} / \mathrm{F})$ e de $Z$ para $F(Z / F)$. Após, utilizando o método da Mínima Curvatura são construídos os mapas de $\mathrm{H}, \mathrm{Z}, \mathrm{F}, \mathrm{H} / \mathrm{F}$ e $\mathrm{Z} / \mathrm{F}$ para cada ano de interesse. Posteriormente, os mapas gerados são analisados e interpretados.

\section{Resultados}

Os valores de H, Z e F para os anos de 1900 e 2015 estão apresentados na Figura 2. Os valores de $\mathrm{H}$, em 1900, variavam entre $23.500 \mathrm{nT}$ e $27.000 \mathrm{nT}$. Em 2015, são observados valores de $\mathrm{H}$ entre $\sim 15.000 \mathrm{nT}$ e 22.500 nT. Isto é, entre 1900 e 2015 houve uma redução de $\sim 36 \%$ no valor mínimo observado e uma redução de $\sim 17 \%$ no valor máximo observado. Para a componente $Z$, considerando os valores em módulo, em 1900 o mínimo observado era de 2.000 nT enquanto o máximo era de $14.000 \mathrm{nT}$. Em 2015, o mínimo era de $\sim 7.500 \mathrm{nT}$ e o máximo era de 18.000 nT. Nestes 115 anos, o valor mínimo aumentou quase quatro vezes enquanto 0 valor máximo aumentou $\sim 29 \%$. Na intensidade total, o valor mínimo observado em 1900 era de $25.400 \mathrm{nT}$ e o valor máximo era de $29.500 \mathrm{nT}$. Em 2015, o mínimo de $\mathrm{F}$ era de 22.300 nT e o máximo era de $24.300 \mathrm{nT}$. Em termos percentuais, o valor mínimo diminuiu $\sim 12 \%$ entre 1900 e 2015 enquanto que no valor máximo é observada uma redução de $\sim 18 \%$.
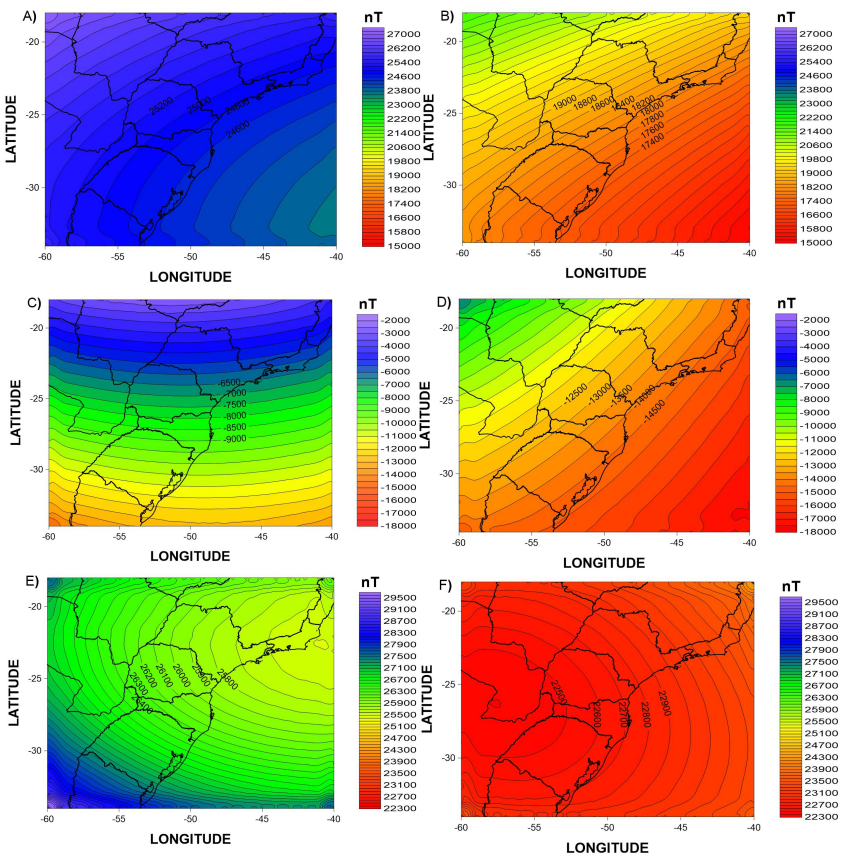

Figura 2- Intensidade horizontal para o ano de 1900 (A) e para o ano de 2015 (B). Intensidade vertical para o ano de 1900 (C) e para o ano de 2015 (D). Intensidade total para o ano de 1900 (E) e para o ano de 2015 (F).

A razão entre a componente horizontal e o campo total é apresentada nos mapas da Figura 3. Para o ano de 1900, a razão $\mathrm{H} / \mathrm{F}$ era alta para praticamente toda a área investigada, com valores geralmente superiores a 0,9, indicando que $\mathrm{H}$ tinha grande importância para a intensidade total. Em 1940, os valores $\mathrm{H} / \mathrm{F}$ menores que 0,9 já ocupam uma área maior que aquela ocupada em 1900. No ano de 1980, a área ocupada por razões $H / F$ menores que 0,9 já ocupa praticamente metade de toda a região investigada. Em 2015, quase que toda a região investigada já é caracterizada por valores de $\mathrm{H} / \mathrm{F}$ menores que 0,9 . Além disso, valores de $H / F$ inferiores a 0,7 são observados no sudeste da área de estudo. Estes resultados indicam que a importância da componente horizontal para a intensidade total vem diminuindo ao longo do último século. 

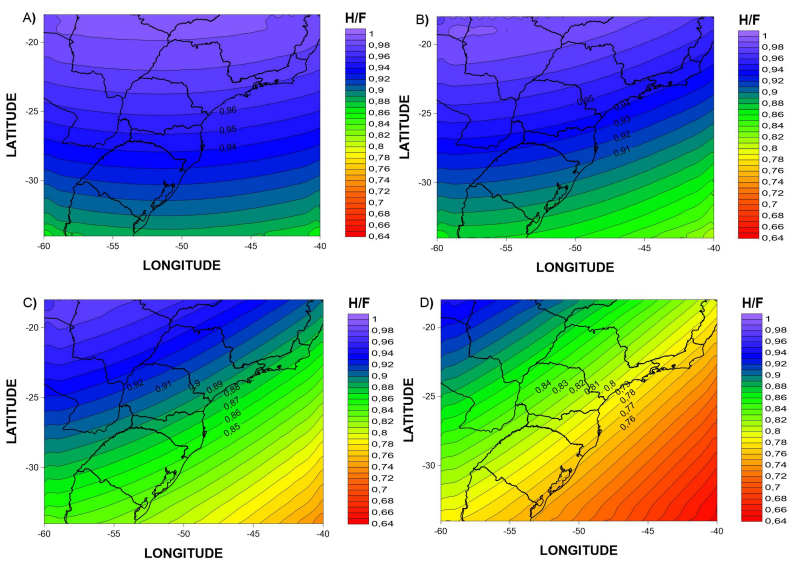

Figura 3- Mapas de razão H/F para os anos de 1900 (A), 1940 (B), 1980 (C) e 2015 (D).

A razão entre a intensidade vertical e a intensidade total está apresentada nos mapas da Figura 4. Em 1900, os valores de Z/F variam entre 0,06 e 0,52. Para 1940, a razão Z/F começa a apresentar aumento, sendo os maiores valores obtidos de 0,58 . Mesmo assim, valores de Z/F menores do que 0,3 ainda cobrem uma parcela significativa da região de estudo. No ano de 1980 , os valores de Z/F maiores do que 0,4 já cobriam mais da metade da região investigada. Em 2015, os valores de $\mathrm{Z} / \mathrm{F}$ aumentaram ainda mais, atingindo o valor 0,78 no sudeste da região investigada.
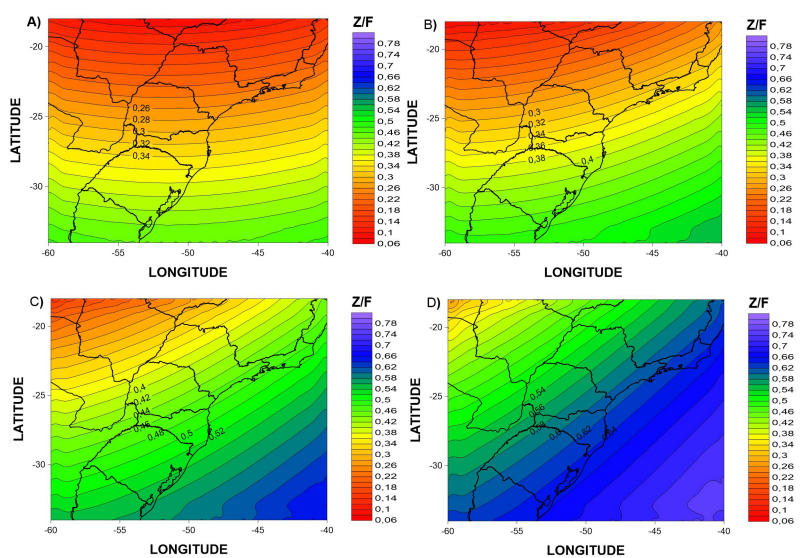

Figura 4- Mapas das razões Z/F para os anos de 1900 (A), 1940 (B), 1980 (C) e 2015 (D).

\section{Conclusão}

Os resultados obtidos para região influenciada pela AMAS mostram uma tendência de decréscimo na intensidade total do campo geomagnético no período entre 1900 e 2015. Esta tendência de decréscimo também é observada na intensidade horizontal. Por outro lado, a intensidade vertical apresenta uma tendência de aumento de valores em módulo ao longo do tempo. Os resultados da razão $\mathrm{H} / \mathrm{F}$ mostram que a contribuição relativa da componente horizontal para o campo total vem diminuindo durante o último século. Por outro lado, os resultados de Z/F, indicam que a contribuição da intensidade vertical para o campo total vem aumentando ao longo dos anos.

O comportamento de $\mathrm{F}, \mathrm{H}, \mathrm{Z}, \mathrm{H} / \mathrm{F}$ e $\mathrm{Z} / \mathrm{F}$ pode estar relacionado ao aumento da influência da AMAS na região. Se a tendência de forte decréscimo nos valores $\mathrm{H}$ acompanhada de decréscimo na contribuição de $\mathrm{H}$ para $\mathrm{F}$ persistir nas próximas décadas, os efeitos indesejáveis associados à PECs tendem a se intensificar na região estudada. Em trabalhos futuros, pretende-se comparar os mapas de razões $\mathrm{H} / \mathrm{F}$ e $\mathrm{Z} / \mathrm{F}$ com os mapas de fluxo de PEC, para identificar possíveis relações entre os mesmos.

\section{Agradecimentos}

Os autores agradecem a UNIPAMPA pelo apoio institucional. Barbara B. Caprara agradece à UNIPAMPA pela concessão de bolsa de Iniciação Científica PDA. Matheus F. da Cruz e Giulia R. Herdies agradecem ao CNPq pela concessão de bolsa de Iniciação Científica PIBIC. Everton Frigo agradece ao CNPq pela concessão de apoio financeiro (Edital Universal 01/2016, processo 429068/2016-6).

\section{Referências}

Campbell, W. H., 2003. Introduction to Geomagnetic Fields. 2.ed. Cambridge University Press. 337 pp.

Grigorian, O. R., Romashova, V. V., and Petrov, A. N., 2008. SAA drift: Experimental Results, Adv. Space Res., $41,76-78$.

Hartmann, G. A.; Pacca, I. G., 2009. Time evolution of the South Atlantic Magnetic Anomaly. Anais da Academica Brasileira de Ciências, v. 81, p. 243-255.

Merrill, R.T., McElhinny, M.W., McFadden, P.L., 1998. The magnetic field of the Earth: Paleomagnetism, the core, and the deep mantle, Vol. 63 (International Geophysics Series.), Elsevier, Academic Press, Amsterdam.

Smart, D. F.; Shea, M. A., 2009. Fifty years of progress in geomagnetic cutoff rigidity determinations. Elsevier Ltd. on behalf of COSPAR, v. 44, p. 1107-1123. 